\title{
Laparoscopy or laparotomy? A comparison of 240 patients with early-stage endometrial cancer
}

\author{
Alessandro Santi · Annette Kuhn · Thomas Gyr • \\ Markus Eberhard · Silke Johann · Andreas R. Günthert • \\ Michael D. Mueller
}

Received: 20 August 2008/Accepted: 20 May 2009/Published online: 16 June 2009

(C) Springer Science+Business Media, LLC 2009

\begin{abstract}
Background This study aimed to compare the safety and efficacy of laparoscopy and laparotomy in the surgical treatment of early endometrial cancer, especially in obese women.

Methods The results obtained after laparoscopic surgical treatment of early endometrial cancer (International Federation of Gynecology and Obstetrics (FIGO) stage 1 or 2) in patients between 1996 and 2007 were compared with an age- and tumour-matched historical group of patients treated with laparotomy between 1988 and 1996. All the patients underwent hysterectomy, bilateral salpingo-oophorectomy, and pelvic \pm paraaortic lymphadenectomy.

Results Both groups included 120 patients with a preoperative diagnosis of early endometrial cancer. The postoperative diagnosis was endometrial cancer stage 1 or 2 for $89 \%$ of the cases in both groups. The mean operating time was $170 \mathrm{~min}$ for the laparotomy group compared with $178 \mathrm{~min}$ for the laparoscopy group (nonsignificant difference). The estimated intraoperative blood loss was significantly greater in the laparotomy group, and the hospital stay was significantly shorter in the laparoscopy group.
\end{abstract}

A. Santi · A. Kuhn $(\bowtie) \cdot$ S. Johann · A. R. Günthert .

M. D. Mueller

Department of Obstetrics and Gynecology, Inselspital,

Bern University Hospital and University of Bern,

Effingerstrasse 102, CH 3012 Bern, Switzerland

e-mail: annette.kuhn@insel.ch

T. Gyr

Reparto di ginecologia ed ostetricia, Ospedale Regionale di

Lugano, Lugano, Switzerland

M. Eberhard

Abteilung für Gynäkologie und Geburtshilfe,

Kantonsspital Schaffhausen, Schaffhausen, Switzerland
Conclusions The results show that early endometrial cancer can be treated effectively by laparoscopy. Because of this study's retrospective design, the results should be interpreted with caution. However, the advantages of this method for obese patients are evident. The age and weight of these patients should not be used as a contraindication for laparoscopy.

Keywords Gynecology and obstetrics · Gynae . Cancer - Obesity

Endometrial cancer is the most common gynecologic malignancy in the United Stated, with approximately 40,000 new cases and 7,400 deaths attributed to this disease annually [1]. More than 1 in 20 female cancers in Europe are endometrial cancer, with increasing trends particularly among postmenopausal women in many countries [2]. Changes in reproductive behavior, obesity, and hormone replacement therapy may partially account for the increase. Whereas obesity may place a patient at increased risk for medical comorbidities including diabetes, cardiovascular diseases, and osteoarthritis, it also is a major risk factor for endometrial cancer. A recent study reported that $68 \%$ of women with early-stage endometrial cancer are obese [3].

The majority of endometrial cancer is without any clinical or histologic sign of extrauterine cancer spread at an early stage. Regional lymph nodes are the most common site of occult metastatic disease in apparent early-stage cancers.

Although endometrial cancer treatment often is via a multimethod approach, the primary step in individualizing a woman's cancer care is surgery, and complete staging 
includes abdominal exploration, pelvic peritoneal cytology, hysterectomy, bilateral salpingo-oophorectomy and pelvic and aortic selective lymphadenectomy. Selective lymphadenectomy is necessary for individualization of adjuvant therapy.

In the past decade, different studies have shown that laparoscopic treatment of early-stage endometrial cancer is an effective and economically efficient alternative to classical laparotomy $[4,5]$. The advantages of laparoscopy demonstrated in other surgical interventions (e.g. shorter hospital stay, quicker recovery, and lower risk of thromboembolic complications and postoperative infections) have already been confirmed for laparoscopic treatment of endometrial cancer [6, 7]. However, patients with endometrial cancer often are both obese and elderly, conditions that make the laparoscopic approach more difficult $[8,9]$.

Although findings have shown laparoscopy to be feasible and reproducible, it is associated with a steep learning curve, most notably with regard to lymphadenectomy. In addition, obesity and morbid obesity may increase the technical issues encountered in this patient population.

The current study aimed to investigate the safety and efficacy of laparoscopy compared with laparotomy for the surgical treatment of early endometrial cancer and to analyze whether obesity is a contraindication for laparoscopic lymphadenectomy.

\section{Patients and methods}

The charts of all patients with clinical early endometrial cancer who underwent surgery between 1988 and 2007 were analyzed retrospectively. The inclusion criterion specified a clinical diagnosis of endometrial cancer stage 1 or 2 according to the International Federation of Gynecology and Obstetrics (FIGO) classification [10], which means that patients preoperatively considered to have stage 1 or 2 disease were included in the study. Patients with a previous malignancy and those who had undergone a vaginal hysterectomy in the past were excluded.

A total of 120 patients were treated consecutively by laparotomy from 1988 to 1996 and 120 patients by laparoscopy from 1996 to 2007. Both techniques included peritoneal washing, hysterectomy, bilateral salpingooophorectomy, and pelvic \pm paraaortic lymphadenectomy.

In the laparoscopy group, a total laparoscopic hysterectomy was performed using a $30^{\circ}$ laparoscope and a uterine manipulator after coagulation of the fallopian tubes if necessary. The uterus and the lymph nodes were evacuated vaginally, with the lymph nodes covered in an Endobag (Endo Pouch, Ethikon, Guaynabo, PR, USA) before removal. All the operations were performed by four gynecologic oncologists.
The patients in the laparoscopy group were age- and tumor-matched with the laparotomy group. Tumor matching included tumor stage and histology with grading. Body mass index (BMI) and operative outcome (operating time, blood loss, number of recovered lymph nodes, length of hospital stay) were comparable between the two groups. For statistical evaluation, a $t$-test was applied using GraphPad Prism version 4.00 for Windows (GraphPad Software, San Diego, CA, USA). Alpha was set at 0.05 as a cutoff value to avoid type 1 error.

\section{Results}

The data for 240 patients were analyzed, which resulted in 120 patients each in the laparoscopy and laparotomy groups. The median age of the patients was 63 years (range, 37-83 years) in the laparotomy group (group 1) and 62 years (range, 40-91 years) in the laparoscopy group (group 2).

Table 1 shows the BMI, intraoperative blood loss, and number of recovered lymph nodes. The intraoperative blood loss was significantly greater in the laparotomy group, with seven patients requiring transfusion. The BMI was not significantly different between the two groups.

Lymph node metastases were confirmed in $5.8 \%$ of group 1 compared with $6.7 \%$ of group 2 (nonsignificant difference). The hospital stay was significantly longer in group $1(6 \pm 4$ days $)$ than in group $2(13.1 \pm 3.7$ days $)$ $(p<0.001)$.

Although the analyzed period was the one in which laparoscopy was introduced for this indication, no major complications were noted. After laparoscopy, one patient experienced a paralysis of the obturator nerve but was recovered completely after 3 months. Another patient

Table 1 Body mass index (BMI), blood loss, number of recovered lymph nodes, and hospital stay

\begin{tabular}{lccl}
\hline & $\begin{array}{l}\text { Group 1 } \\
\text { (laparotomy) }\end{array}$ & $\begin{array}{l}\text { Group 2 } \\
\text { (laparoscopy) }\end{array}$ & $p$-Value \\
\hline FIGO 1 $+2(\%)$ & 89 & 89 & NS \\
FIGO 3 (\%) & 11 & 10.5 & NS \\
FIGO 4 (\%) & 0 & 0.5 & NS \\
Operating time (min) & 150 & 165 & NS \\
Blood loss (ml) & 580 & 240 & $<0.001$ \\
BMI (kg/m ${ }^{2}$ ) & 29.6 & 31.6 & NS \\
$\begin{array}{l}\text { Recovered lymph } \\
\text { nodes }(n)\end{array}$ & 18.1 & 21.2 & $<0.05$ \\
$\begin{array}{l}\text { Postoperative hospital } \\
\text { stay (days) }\end{array}$ & 13.2 & 5.6 & $<0.001$ \\
\hline
\end{tabular}

FIGO International Federation of Gynecology and Obstetrics staging; $N S$ not significant 


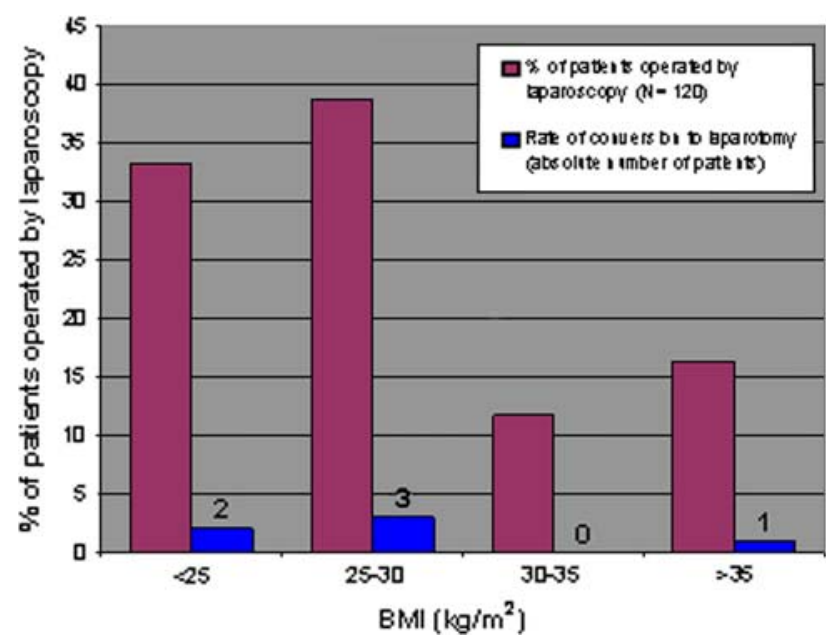

Fig. 1 Patients operated by laparoscopy (\%) and BMI with the rate of conversion to laparotomy

experienced a symptomatic lymphocele after laparoscopy, which required laparoscopic marsupialization after three unsuccessful trials of drainage that was ultrasonographically guided. In both groups, intraoperative staging showed an advanced FIGO stage $(>2)$ in $11 \%$ of cases.

Figure 1 shows the rate of conversion from laparoscopy to laparotomy depending on the BMI. In group 1,31 $(25.8 \%)$ of the patients had a BMI higher than $30 \mathrm{~kg} / \mathrm{m}^{2}$, and $18(15 \%)$ of these had a BMI exceeding $35 \mathrm{~kg} / \mathrm{m}^{2}$, with a maximal BMI of $50.2 \mathrm{~kg} / \mathrm{m}^{2}$. When patients with a BMI higher than $30 \mathrm{~kg} / \mathrm{m}^{2}$ were analyzed separately, we could not see a correlation between BMI and rate of conversion to laparotomy, between BMI and blood loss, or between BMI and the number of recovered lymph nodes. The operating time for the patients with a BMI greater than $30 \mathrm{~kg} / \mathrm{m}^{2}$ was statistically longer than for those with a BMI less than $30 \mathrm{~kg} / \mathrm{m}^{2}$ (170 vs $\left.202 \mathrm{~min} ; p=0.0184\right)$.

A conversion to laparotomy was necessary in $6(5 \%)$ of the 120 cases managed by laparoscopy. For no patient was the conversion to laparotomy due to obesity (Fig. 1). For five patients, conversion was performed because of hemorrhage. In one case, the conversion was performed after evidence of intraperitoneal tumor dissemination.

\section{Discussion}

As the prevalence of overweight and obese women continues to rise, an increased incidence of endometrial cancer may be expected in this patient population. In addition to an increased risk of endometrial cancer, these patients are at an increased risk for medical comorbidities including diabetes and cardiovascular diseases. Consequently, interest has focused on identifying surgical techniques that may provide adequate surgical treatment for this patient population while minimizing surgical morbidity and mortality.

Increasing numbers of gynecologic oncologists are suggesting laparoscopy as an alternative for their patients with endometrial cancer [11-14]. Concerns about adequacy of staging, a lack of long-term survival and recurrence data, the learning curve, and prolonged operative times have prevented the wide acceptance of laparoscopy. Because many gynecologists believe obesity precludes laparoscopic staging, they do not offer minimally invasive surgery to obese patients. However, despite the limited number of studies supporting the advantages of laparoscopy, growing evidence shows that the minimally invasive procedure offers adequacy of staging, reasonable operating times, less blood loss, and a shorter hospital stay compared with laparotomy [15-17].

Eisenhauer et al. [17] compared the outcomes of obese women undergoing various surgical procedures for the management of endometrial carcinoma. These authors concluded that laparoscopic staging and panniculectomy at the time of laparotomy were associated with a better lymph node count and a lower rate of incisional complications than laparotomy alone. In their study, the length of hospital stay was 3 days for the patients treated laparoscopically.

The current study shows a significantly improved length of hospital stay for the women in the laparoscopic group. However, the hospital stay of 6 days this is longer than in the aforementioned study [17]. The length of stay may be due to the postoperative pathway at the time of the study, which involved prolonged Foley catheter use and diet restrictions that that have been changed in the meantime.

Several articles are available regarding the laparoscopic treatment of early endometrial cancer $[8,9,11]$. A randomized study by Zullo et al. [18] has confirmed the laparoscopic approach to be a feasible and safe procedure for the treatment of stage 1 endometrial cancer. These results also are supported by our findings, although the main weak point of our study probably is the retrospective design, which we need to stress explicitly. This makes the current paper more open to bias and may explain why our results are rather equivalent, involving possible difficulties with data collection, although the latter actually was not a problem in our series.

Indeed, laparoscopic surgery currently is replacing classical laparotomy in the management of early-stage endometrial cancer. Our study and previous publications that analyzed perioperative morbidity or validated the laparoscopic method of examining the safety and extending oncologic staging, including the number of harvested lymph nodes during the procedure, have demonstrated results similar to those for laparotomy. Additionally, other advantages such as a shorter hospital stay and immediate 
increased postoperative quality of life are clearly in favor of the laparoscopic approach $[5,18]$.

However, different authors report limitations of laparoscopy for patients with a high BMI [6, 19], which would be a major drawback of the technique, especially considering that these patients are at risk for the development of endometrial cancer. Our data support the contrary, showing that endometrial cancer in severely obese women can be managed safely by laparoscopy. Neither the conversion rate nor the complication rate was higher for this specific group.

In the laparoscopic group, $31(25.8 \%)$ patients had a BMI higher than $30 \mathrm{~kg} / \mathrm{m}^{2}$, and $18(15 \%)$ of these had a BMI exceeding $35 \mathrm{~kg} / \mathrm{m}^{2}$, with a maximal BMI of $50.2 \mathrm{~kg} /$ $\mathrm{m}^{2}$ (Fig. 1). A conversion to laparotomy was necessary in 6 $(5.0 \%)$ of the 120 cases managed by laparoscopy. In none of these cases was the conversion to laparotomy due to obesity. It was due to hemorrhage in five cases and tumor stage in one case. Although these indications for laparoscopy were new, no major complications occurred, which supports the safety of the laparoscopic approach.

These results need to be interpreted with caution because of the study's retrospective design. In a prospective study, we might have been able to identify minor complications more easily. Minor complications may be underreported because they were not considered sufficiently important to be noted explicitly or because patients with minor complications may have contacted their general practitioner or referring gynecologist.

A retrospective approach may imply difficulties in data collection. However, because we examined clearly defined outcome measures, this was not a problem we consider very significant.

Four different surgeons performed the interventions, which may have led to a variation in outcomes. However, because all the surgeons used the same technique recommended by the Swiss group of endoscopic surgeons, the surgical techniques are comparable. Additionally, it was possible to use validated quality-of-life and pain questionnaires for the immediate postoperative and recovery period to determine patient preferences for either approach, particularly in this elderly patient group. This subject will be addressed in future studies.

The blood loss in the group of patients with a BMI exceeding $30 \mathrm{~kg} / \mathrm{m}^{2}(168 \mathrm{ml})$ was not statistically higher than in the group with a BMI lower than $30 \mathrm{~kg} / \mathrm{m}^{2}$ $(190 \mathrm{ml})$. The median number of recovered lymph nodes $(n=21)$ demonstrates sufficient surgical staging [20] that provided accurate information on the stage of disease. The key to sufficient surgical staging is a team fully trained in the management of endometrial cancer because increased surgical experience improves the number of recovered lymph nodes, as shown by Barakat et al. [21].
Our results and recent data suggest the comparability of laparotomy and laparoscopy in terms of oncologic safety for the treatment of early stage endometrial cancer $[5,22$, 23]. A recent prospective trial [24] found a higher vaginal cuff recurrence rate for patients treated with laparoscopic surgery than for those treated with a laparotomy approach, although this difference did not reach statistical significance. In this regard, the latter finding seems to be in agreement with a previous study [25], although there is no unanimous consensus [25-27].

Because the surgeons participating in this study were already experienced in laparoscopic surgery, our results demonstrate that with appropriate experience in endoscopic and oncological surgery, endometrial cancer can be treated by laparoscopy without the need for a long learning curve [28]. When our first 120 consecutive cases of laparoscopically treated patients were compared with an age- and tumor-matched historical group, the operation time and the number of recovered lymph nodes were similar in the two groups.

The current study supports the laparoscopic approach for endometrial cancer in elderly and obese patients. Future prospective studies will include validated pain and qualityof-life scores as well as oncologic and cosmetic outcomes.

\section{References}

1. Jemal A, Sieghel R (2007) Cancer statistics 2007. CA Cancer J Clin 57:43-66

2. Bray F, Dos Santos Silva I, Moller H, Weiderpass E (2005) Endometrial cancer incidence trends in Europe: underlying determinants and prospects for prevention. Cancer Epidemiol Biomarkers Prev 14:1132-1142

3. von Grueningen VE, Gil KM, Frasure HE (2005) Complementary medicine use, diet, and exercise in endometrial cancer survivors. J Cancer Integ Med 3:13-18

4. Childers JM, Surwit EA (1992) Combined laparoscopic and vaginal surgery for the management of two cases of stage 1 endometrial cancer. Gynecol Oncol 45:46-51

5. Spirtos NM, Schlaerth JB, Gross GM, Spirtos TW, Schlaerth AC, Ballon SC (1996) Cost and quality-of-life analyses of surgery for early endometrial cancer: laparotomy versus laparoscopy. Am J Obstet Gynecol 174:1795-1799

6. Ghezzi F, Cromi A, Bergamini V, Uccella S, Beretta P, Franchi $M$ et al (2006) Laparoscopic management of endometrial cancer in nonobese and obese women: a consecutive series. J Minim Invasive Gynecol 13:269-275

7. Yu CK, Cutner A, Mould T, Olaitan A (2005) Total laparoscopic hysterectomy as a primary surgical treatment for endometrial cancer in morbidly obese women. BJOG 112:115-117

8. Scribner DR Jr, Walker JL, Johnson GA, McMeekin SD, Gold MA, Mannel RS (2001) Surgical management of early-stage endometrial cancer in the elderly: is laparoscopy feasible? Gynecol Oncol 83:563-568

9. Obermair A, Manolitsas TP, Leung Y, Hammond IG, McCartney AJ (2005) Total laparoscopic hysterectomy versus total abdominal hysterectomy for obese women with endometrial cancer. Int $\mathbf{J}$ Gynaecol Cancer 15:319-324 
10. FIGO (1989) Corpus cancer staging. Int J Gynecol Obstet 28:190

11. Boike G, Lurain J, Burke J (1994) A comparison of laparoscopic management for endometrial cancer with traditional laparotomy. Gynecol Oncol 52:195

12. Magrina JF, Serrano L, Cornella JL (1995) Laparoscopic lymphadenectomy and radical or modified radical vaginal hysterectomy for endometrial or cervical carcinoma: preliminary experiences. J Gynecol Surg 11:147-151

13. Spirtos NM, Schlaerth JB, Spirtos TW (1995) Laparoscopic bilateral pelvic and paraaortic lymph node sampling: an evolving technique. Am J Obstet Gynecol 173:105-111

14. Gemignani ML, Curtin JP, Zelmanovich J (1999) Laparoscopicassisted vaginal hysterectomy for endometrial cancer: clinical outcomes and hospital charges. Gynecol Oncol 73:5-11

15. Eltabbakh GH, Mount SL (2006) Laparoscopic surgery does not increase the positive peritoneal cytology among women with endometrial carcinoma. Gynecol Oncol 100:361-364

16. Scribner DR, Walker JL, Johnson GA (2002) Laparoscopic pelvic and paraaortic lymph node dissection in the obese. Gynecol Oncol 84:426-430

17. Eisenhauer EL, Wyppych KA, Mehrara BJ (2007) Comparing surgical outcomes in obese women undergoing laparotomy, laparoscopy, or laparotomy with panniculectomy for the staging of uterine malignancy. Am surg Oncol 14:2384-2391

18. Zullo F, Palomba S, Russo T, Falbo A, Costantino M, Tolino A et al (2005) A prospective randomized comparison between laparoscopic and laparotomic approaches in women with early stage endometrial cancer: a focus on the quality of life. Am J Obstet Gynecol 193:1344-1352

19. O'Hanlan KA, Dibble SL, Fisher DT (2006) Total laparoscopic hysterectomy for uterine pathology: impact of body mass index on outcomes. Gynecol Oncol 103:938-941

20. Smith DC, Macdonald OK, Lee CM, Gaffney DK (2008) Survival impact of lymph node dissection in endometrial adenocarcinoma: a surveillance, epidemiology and end results analysis. Int J Gynecol Cancer 18(2):255-261

21. Barakat RR, Lev G, Hummer AJ, Sonoda Y, Chi DS, Alektiar $\mathrm{KM}$ et al (2007) Twelve-year experience in the management of endometrial cancer: a change in surgical and postoperative radiation approaches. Gynecol Oncol 105:150-156

22. Frigerio L, Gallo A, Ghezzi F, Trezzi G, Lussana M, Franchi M (2006) Laparoscopic-assisted vaginal hysterectomy versus abdominal hysterectomy in endometrial cancer. Int J Gynaecol Obstet 93:209-213

23. Tozzi R, Malur S, Koehler C, Schneider A (2005) Laparoscopy versus laparotomy in endometrial cancer: first analysis of survival of a randomized prospective study. J Minim Invasive Gynecol 12:130-136

24. Zullo F, Palomba S, Falbo A, Russo T, Mocciaro R, Tartaglia E, Piersandro T, Mastrantonio P (2009) Laparoscopic surgery vs laparotomy: long-term data of a randomized controlled trial. Am J Obstet Gynecol 200:2961-2969

25. Chu CS, Randall TC, Bandera CA, Rubin SC (2003) Vaginal cuff recurrence of endometrial cancer treated by laparoscopic assisted vaginal hysterectomy. Gynecol Oncol 88:62-65

26. Malur S, Possover Michels W, Schneider A (2001) Laparoscopic versus abdominal surgery in patients with endometrial cancer: a prospective randomized trial. Gynecol Oncol 80:239-244

27. Fram KM (2002) Laparoscopically assisted vaginal hysterectomy versus abdominal hysterectomy in stage I endometrial cancer. Int J Gynecol Cancer 12:57-61

28. Fleisch MC, Newton J, Steinmetz I, Whitehair J, Hallum A, Hatch KD (2007) Learning and teaching advanced laparoscopic procedures: do alternating trainees impair a laparoscopic surgeon's learning curve? J Minim Invasive Gynecol 14:293-299 